Article

\title{
Changes of gut microbiota in FAP and UC patients in Mediter- ranean Region of Turkey: an -omic landscape to be discovered
} Abdullah HANTA + $^{1}$, Ahmet Rencuzogullari + $^{2}$, Ibrahim BOGA ${ }^{1,3}$, Ismail Cem ERAY ${ }^{2}$ and Atil Bisgin 1,3*

1 Cukurova University, AGENTEM (Adana Genetic Diseases Diagnosis and Treatment Center), Adana, Turkey; abdullahhanta@outlook.com

2 Cukurova University, Faculty of Medicine, Department of Surgical Oncology, Adana, Turkey; arencuz@cu.edu.tr; iceray@cu.edu.tr

3 Cukurova University, Faculty of Medicine, Department of Medical Genetics, Adana, Turkey; abisgin@yahoo.com ; ibr.boga@gmail.com

$\dagger \quad$ First two authors AH and AR contributed equally.

Citation: Hanta, A.; Rencuzogullari, A.; Boga, I.; Eray,I.C.; Bisgin,A. Changes of gut microbiota in FAP and UC patients in Mediterranean Region of Turkey: an -omic landscape to be discovered. Genes 2021, 12, x. https://doi.org/10.3390/xxxxx

* Correspondence: abisgin@yahoo.com

Abstract: Inflammatory bowel diseases, familial adenomatous polyposis (FAP) and colorectal cancer $(\mathrm{CRC})$ are associated with alterations of the intestinal microbiota. However, few data are available on the perpetuation of FAP and ulcerative colitis (UC) in relation to microbial dysbiosis. This study evaluated the UC and genetically confirmed FAP patients' gut microbial balance in concordance to clinical outcome. Fecal materials (average mass of $0.54 \mathrm{~g}$ ) were collected from three FAP and five UC patients to compare with healthy individuals as control group. Genomic materials of microbiota were isolated for next generation sequencing of 16S rRNA that was performed by using QIAseq 16S/ITS panel in Illumina Miseq Platform. Data processing and bioinformatics analysis were performed via CLC Genomic Workbench bioinformatics tool. The comparison between FAP, UC and control group revealed an alteration in the intestinal microbial composition. More in details, relative abundance of class levels showed statistical significance differences among FAP, UC and control groups. Our preliminary data focused on the explanation of how dysbiosis can lead to inflammation and drive processes together with host genetic profile that leads to colorectal carcinogenesis.

Keywords: Dysbiosis, Familial adenomatous polyposis, Ulcerative Colitis, $16 \mathrm{~S}$ rRNA, Gut Microbiota, Geographic Populations

\section{Introduction}

Evolution is the core explanation of life that enables us to examine the changes that organisms pass through their generations and the properties they transmit, from a single-celled to multi-celled perspective. In this way, especially the studies of the relationships between microorganisms with each other and with different species has led to the health sciences involve and interact with microbial evolution in a state of necessity. This necessity paves the road to new research fields on human microbiota which are located in 
several different sites of human body such as nasal passages, oral cavity, skin, gastrointestinal tract, and urogenital tract.

The human microbiome is composed of bacteria, archaea, viruses, and eukaryotic microbes which are dispersed in and on body. These microbes have various impacts on hu-man physiology both in health and in diseases. The expanding studies on microbiome favor gut microbiota as a 'real organ' that inhabits on gut mucosa and characterized by fluctuating changes in microbial diversity during the first years of life until an eventual convergence towards an adult microbiota[1,2].

The digestive system contributes to energy, maintenance, development, and reproduction of organism by processing nutrients. Nutrients are broken down into small pieces by digestive enzymes beginning from the mouth, while the necessary digestion and absorption is made with motor and muscle movements in the digestive tract. This process happens in a mutualistic way with the microorganisms in the microbiota hosted in human mucosa by providing additional nutrients that are not subject to endogenous digestive enzymes and by synthesizing amino acids and vitamins to host organism. On the other hand, there are microorganisms that are harmful to the digestive system as well as beneficial organisms. Gut microbiota studies conducted so far have shown that emerging harmful microorganisms and/or imbalance of gut microbiota which referred as "dysbiosis" cause various physiological, metabolic, nutrition, immunological, cardiovascular even psychiatric diseases[3-10].

Most recent developments in molecular genetic techniques reveal that the individuals with colorectal cancer (CRC) also harbour abnormalities in gut microbiome. However, the gut microbiome patients with precancerous lesions including the familial adenomatous polyposis (FAP) and ulcerative colitis (UC) remains largely unknown. The interaction between the colon itself and its environment is complex with population and disease-based variation. Nevertheless, many studies examined the microbiota in cancer aetiology without the important drivers of risk such as FAP and UC with microbiota and population association.

The primary aim of this study, therefore, was to study the microbiome via 16S rRNA/ITS sequencing of the patients who have CRC predisposition syndromes of FAP and UC from the Mediterranean Region of Turkey who have CRC predisposition syndromes of FAP with mutations detected in APC and MUTYH genes and UC, excluding the heterogeneity of diet and other environmental factors in comparison with healthy individuals having similar habits. Further, we also aimed to describe the local ecology of gut microbiome in the Mediterranean coast of Turkey.

\section{Materials and Methods}

Patients

Fecal samples were collected from a total of 12 people, three of whom were considered healthy controls, three of whom were both clinically and genetically diagnosed with FAP, and five were patients diagnosed with UC. Additional peripheral blood samples were collected from all individuals in order to identify the disease related mutations. All the subjects included in the gut microbiome study were living in the Mediterranean region and they are distributed as follows: Healthy controls are from 2 female and 1 male individual with a mean age of 34 years, FAP patients are also from two female and one male patients with a mean age of 39.3 years, and UC patients consist of 3 female and 2 male patients with a mean age of 49.6 years. 
DNA isolation

According to the manufacturer's user manual, the required minimum $0.25 \mathrm{~g}$ of stool sample was collected by the sterile fecal container and weighed, and the excess material was discarded. Average mass of fecal materials w

re $0.54 \mathrm{gr}$ for all samples. Extraction protocol was applied within 1 hour after the samples were taken. Genomic materials of microbiota were isolated via QIAamp PowerFecal DNA kit (QIAGEN, Hilden, Germany, Catalog no. 12830-50) according to manufacturer instructions. DNAs were quantified using the Qubit dsDNA HS (High Sensitivity) Assay Kit (Invitrogen by Thermo Fisher Scientific, Waltham, MA, USA) on a Qubit 3 Fluorometer (Invitrogen).

Additionally, peripheral blood samples of FAP and UC patients who referred to Cukurova University AGENTEM (Adana Genetic Disease, Diagnosis and Treatment Center) were sequenced via MiSeq NGS System (Illumina) through a custom design multigene panel including FAP related genes (APC and MUTYH). The 12 blood samples were isolated from leukocytes using QIAsymphony DSP DNA Midi Kit (Qiagen, Hilden, Germany) according to the manufacturer's instructions. The quality and quantity of the DNA samples was determined by Qubit ${ }^{\mathrm{TM}}$ Fluorometric Quantitation (Thermo Fisher Scientific, Waltham, MA, USA).

Gut Microbiome analysis

Next generation sequencing of 16S rRNA was performed by using QIAseq 16S/ITS panel in Illumina Miseq Platform. Data processing and bioinformatics analysis were performed via CLC Genomic Workbench bioinformatics tool. Read pairs were assembled using clc_assembler from the CLC Genomics Workbench 20.0 analysis package (www.clcbio.com/products/clc-genomics-workbench).

Operational Taxonomic Unit (OTU) clustering and taxonomic analyses were performed using CLC Genomics Workbench v. 20.0 and CLC Microbial Genomics Module (Qiagen). Sequences were first trimmed and merged and then were clustered into OTUs at $97 \%$ sequence similarity level using the Amplicon-Based OTU clustering tool. The most abundant sequences were selected as representative of each cluster and then assigned to a taxonomy level using CLC Microbial Genomics default values and the Greengenes and Silva Databases. 
Mutational analysis

All samples were targeted enriched for APC and MUTYH gene (all exons and exon-intron junctions) with a custom designed multigene panel. Amplicons were labeled with sample specific molecular barcodes for library generation. Finally, next generation sequencing was performed via Illumina MiSeq (Illumina, California, USA) platform with the minimum coverage of 100x. The data sizes of FASTQ files and variant qualities were checked for quality assessments. Bioinformatics analyses were carried out using QCI-A and QCI-I (Qiagen, Hildenberg, Germany). Detected variants were interpreted comparatively with 18 databases. All identified genetic alterations were categorized based on their pathogenicity according to the American College of Medical Genetics (ACMG) criteria.

\section{Results}

The results were examined in two main sections: Genomic results of the host organism and abundance of microbial species located on the gut microbiota of the hosts. The distribution of age, gender, fecal sample weight and fecal DNA concentrations of the samples are shown in Table 1.

Table 1. The distribution of age, gender, fecal sample weight and fecal DNA concentrations.

\begin{tabular}{cccccc}
\hline Sample & Diagnosis & Sex & Age & $\begin{array}{c}\text { Sample } \\
\text { weight } \\
\text { (gr) }\end{array}$ & $\begin{array}{c}\text { DNA } \\
\text { Concentration } \\
\text { (ng/ } \boldsymbol{\mu L})\end{array}$ \\
\hline S1 & Control & Female & 32 & 0.2006 & 19.4 \\
S2 & Control & Female & 33 & 0.4656 & 61 \\
S3 & Control & Male & 37 & 0.3857 & 63.6 \\
S4 & UC & Female & 37 & 0.6594 & 43.4 \\
S5 & UC & Male & 67 & 0.5981 & 57.6 \\
S6 & FAP & Male & 29 & 0.4871 & 16.2 \\
S7 & FAP & Female & 33 & 0.7239 & 11.3 \\
S8 & FAP & Female & 56 & 0.7724 & 1.41 \\
S9 & UC & Male & 64 & 0.7201 & 12.1 \\
S10 & UC & Female & 38 & 0.2161 & 2.62 \\
S11 & UC & Female & 42 & 0.6827 & 36.4 \\
\hline
\end{tabular}

When the means of patients' age, fecal sample weights and fecal DNA concentrations were calculated for the control, FAP and UC groups, respectively, the mean age was 34, 39.3 and 49.6, the mean fecal sample weights were $0.3506,0.6611$ and $0.5753 \mathrm{gr}$, and the mean of fecal DNA concentrations is 48.0, 9.6 and $30.4 \mathrm{ng} / \mu \mathrm{L}$.

One of the APC gene and gut microbiome results from a total of 12 subjects was excluded from the results due to insufficient data available. In two of the remaining eleven samples, the pathogenic variant NM_000038 (APC): c.3921_3925del (p. E1309Ifs*4) was observed as heterozygous at two FAP patients (S6 and S7) while no mutations were detected on others. 
Table 2. Microbial abundance table of class levels compared between FAP, UC, and control group

\begin{tabular}{cccc}
\hline 16bass (Aggregated) & $\begin{array}{c}\text { Control } \\
\text { Abundance }\end{array}$ & $\begin{array}{c}\text { UC } \\
\text { Abundance }\end{array}$ & $\begin{array}{c}\text { FAP } \\
\text { Abundance }\end{array}$ \\
\hline 157Actinobacteria & 6584 & 76880 & 13162 \\
Alpha proteobacteria & 8999 & 2356 & 0 \\
158 Bacilli & 4806 & 197740 & 237684 \\
Bacteroidia & 1324736 & 1374676 & 321973 \\
159ampylobacteria & 0 & 73 & 0 \\
Clostridia & 748894 & 613039 & 897452 \\
160Coriobacteriia & 5684 & 5865 & 13543 \\
Deltaproteobacteria & 4937 & 8 & 0 \\
16Erysipelotrichia & 67878 & 333462 & 28289 \\
Fusobacteriia & 18 & 3745 & 10 \\
G62mmaproteobacteria & 32850 & 1859739 & 269883 \\
163 Halanaerobiia & 270 & 0 & 0 \\
Kiritimatiellae & 7 & 0 & 0 \\
164 Lentisphaeria & 754 & 0 & 0 \\
Melainabacteria & 2011 & 0 & 0 \\
16Elethanobacteria & 656 & 0 & 0 \\
Mollicutes & 29069 & 9 & 49 \\
166 Negativicutes & 57863 & 223068 & 3666 \\
Oligosphaeria & 17 & 0 & 0 \\
168xyphotobacteria & 2 & 115 & 0 \\
Saccharimonadia & 0 & 4836 & 0 \\
168 Stramenopiles & 22 & 0 & 0 \\
Verrucomicrobiae & 3206 & 5733 & 252 \\
\hline 169 & & &
\end{tabular}

Disease perpetuation was correlated with changes in relative abundance at different levels within both FAP and UC. Based on our observation that the FAP has an overall larger effect on gut microbiome than UC compared to healthy individuals. Figure 1 a as a relative abundances bar graphs for the top abundant classes including the top proteobacteria, euryarchaeota methanobacteria, lentisphaerae lentisphaeria, cyanobacteria melainbacteria, fusobacteria fusobacteriia, patesci bacteria, proteobacteria deltaproteobac-teria, verrucomicrobia verrucomicrobiae, proteobacteria alphaproteobacterial, actinobacte-ria coriobacteria and firmucutes, indicates the disturbance and changes in details according to the clinical status. Figure $1 \mathrm{~b}$ also showing the heatmap of relative abundance and distribution of class-based taxonomic units' reads. The color code indicates the relative abundance, ranging from red to black thru low and high abundance.

Thus, microbiota composition and relative abundance were significantly altered with precancerous lesions compared to the healthy individuals with the same diet and habits. 


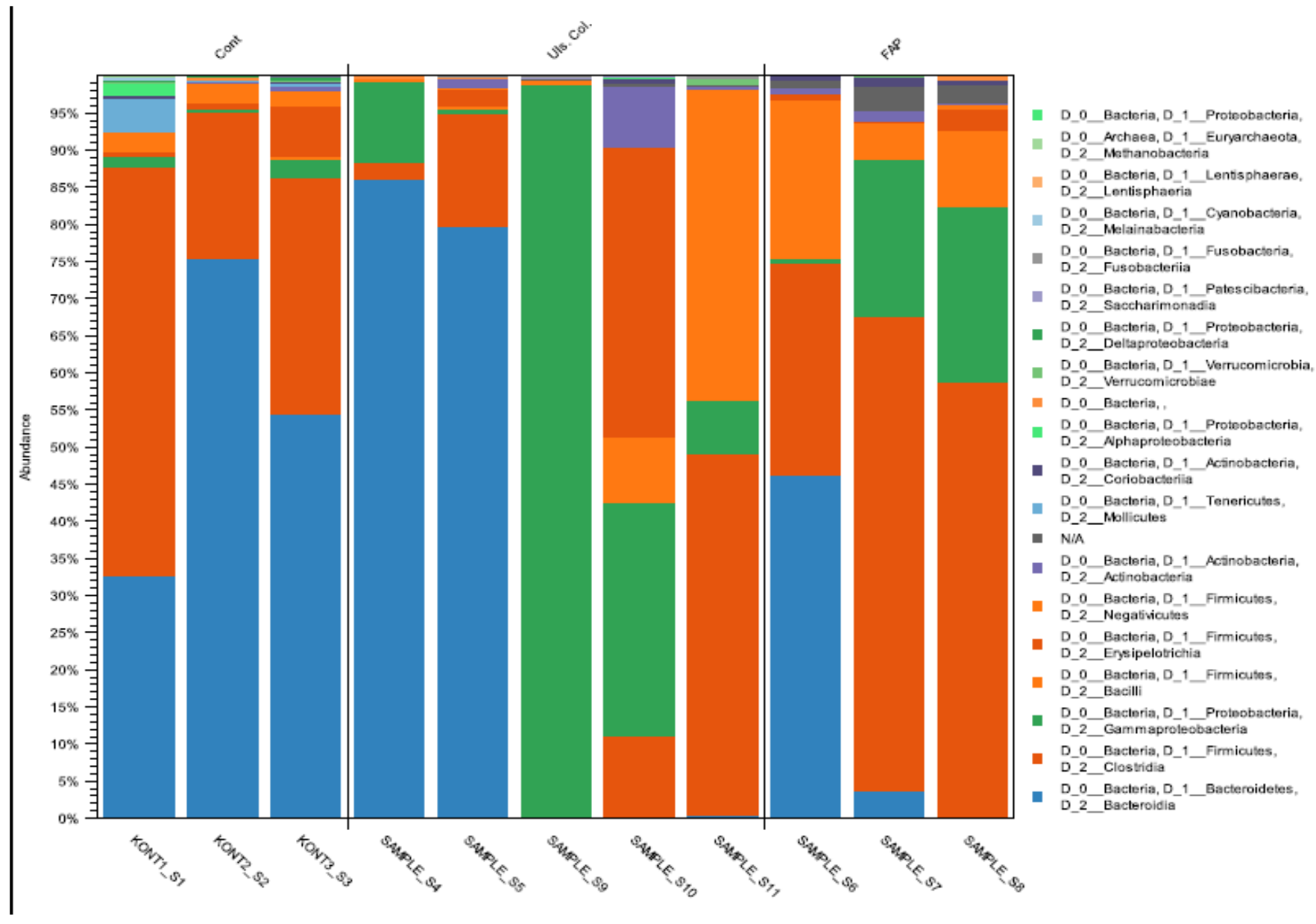

(a) 


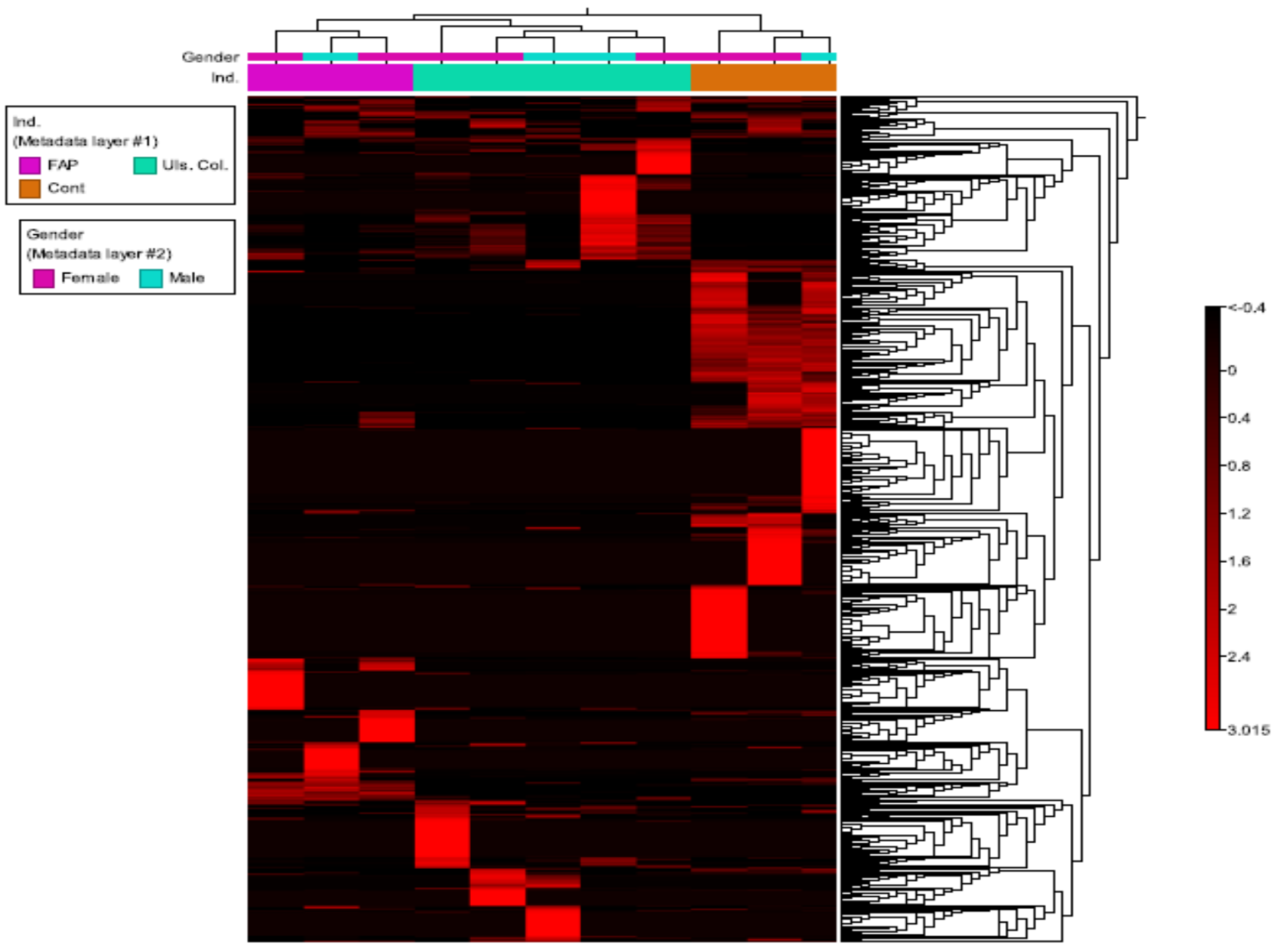

(b)

Figure 1. Relative Abundance of microbial classes (based on total 16S rRNA/ITS sequences) and Heat map graphs based on disease indication and gender metadata of in accordance to Control, UC and FAP groups listed as: (a) Relative Abundance of microbial classes which are proteobacteria, euryarchaeota methanobacteria, lentisphaerae lentisphaeria, cyanobacteria melainbacteria, fusobacteria fusobacteriia, patesci bacteria, proteobacteria deltaproteobacteria, verrucomicrobia verrucomicrobiae, proteobacteria alphaproteobacterial, actinobacteria coriobacteria, firmucutes ; (b) Heat map graphs based on disease indication and gender metadata.

\section{Discussion}

We surveyed the bacterial composition of fecal samples from Mediterranean region of Turkey related to patients with FAP and UC by minimizing the environmental variables including the diet and its relationship with the common genetic mutation related disease status. Our aim was to show the differences between the healthy control group and patients with CRC predisposition syndromes of FAP and UC seems to have achieved its purpose in the light of the results we mentioned above. In addition to the difficulty of directly comparing gut microbiome of CRC predisposition syndromes of FAP and UC, our study also uses APC gene variants to empower our CRC predisposition diagnosis.

The NM_000038(APC): c.3927_3931del (p. E1309Ifs*4) pathogenic variant in the APC gene was detected as heterozygous in two of eight patients as a result of the next generation sequencing. These variants found are important in terms of their contribution to the specificity of our study in the Mediterranean region, since they are also seen in another FAP study belonging to the Mediterranean region[11]. 
We contextualize our results with taxa-level meta-analysis of from previous studies, which identified bacterial phylum that primarily associate with CRC (fusobacteria, actinobacteria Cori bacteria, deltaproteobacteria)[12-14]. It is likely that not all associations in this study are related to specific disease related conditions, but our approach is supported by the results of dysbiosis. Only few studies have shown difference in the composition of gut microbiome between the local populations such as North American and Scandinavian countries. However, all the Mediterranean countries especially the regions in the Mediterranean coast are composed of historically and genetically related populations with similar lifestyles and traditions, which may determine the difference of individuals from other countries.

In order to further investigate relationships between taxonomic groups and other environmental such as diet or disease related gene variants, the correlation coefficiency must be calculated between taxonomic groups and other parameters in larger study groups. However, there is still lack of knowledge about the regional based microbiome data as well as the national genomic studies. After all larger studies in relation to population-based genomics data will help us to model a risk index for CRC perpetuation enabling the detection of associations, even to the level of individual taxa.

Author Contributions: “Conceptualization, A.B.; methodology, A.H.; investigation, A.H. and I.B.; resources, A.R. and I.C.E; writing-original draft preparation, A.H.; writing-review and editing, A.B.; visualization, A.H. and I.B. All authors have read and agreed to the published version of the manuscript."

Funding: This research received no external funding.

Institutional Review Board Statement: The research was reviewed and approved by the Cukurova University in compliance with Declaration Helsinki11.

Informed Consent Statement: Informed consent was obtained from all patients or from their parents enrolled in the study.

Data Availability Statement: The datasets used and/or analyzed during the current study are available from the corresponding author upon reasonable request.

Acknowledgments: We would like to thank to all our patients for their enrollment and the technopark initiative InfoGenom-AB for its contribution.

Conflicts of Interest: The authors declare that they have no conflict of interests.

\section{References}

1. DiGiulio, D.B.; Romero, R.; Amogan, H.P.; Kusanovic, J.P.; Bik, E.M.; Gotsch, F.; Kim, C.J.; Erez, O.; Edwin, S.; Relman, D.A. Microbial Prevalence, Diversity and Abundance in Amniotic Fluid During Preterm Labor: A Molecular and Culture-Based Investigation. PLoS One 2008, 3, doi:10.1371/journal.pone.0003056.

2. Mulder, I.E.; Schmidt, B.; Lewis, M.; Delday, M.; Stokes, C.R.; Bailey, M.; Aminov, R.I.; Gill, B.P.; Pluske, J.R.; Mayer, C.-D.; et al. Restricting Microbial Exposure in Early Life Negates the Immune Benefits Associated with Gut Colonization in Environments of High Microbial Diversity. PLoS One 2011, 6, doi:10.1371/journal.pone.0028279.

3. Sekirov, I.; Russell, S.L.; Antunes, L.C.M.; Finlay, B.B. Gut Microbiota in Health and Disease. Physiological Reviews 2010, 90, 859-904, doi:10.1152/physrev.00045.2009.

4. Wang, Z.; Klipfell, E.; Bennett, B.J.; Koeth, R.; Levison, B.S.; DuGar, B.; Feldstein, A.E.; Britt, E.B.; Fu, X.; Chung, Y.-M.; et al.

Gut flora metabolism of phosphatidylcholine promotes cardiovascular disease. Nature 2011, 472, 57-63, 
5. Le Chatelier, E.; Nielsen, T.; Qin, J.; Prifti, E.; Hildebrand, F.; Falony, G.; Almeida, M.; Arumugam, M.; Batto, J.-M.; Kennedy, S.; et al. Richness of human gut microbiome correlates with metabolic markers. Nature 2013, 500, 541-546, doi:10.1038/nature12506.

6. Koeth, R.A.; Wang, Z.; Levison, B.S.; Buffa, J.A.; Org, E.; Sheehy, B.T.; Britt, E.B.; Fu, X.; Wu, Y.; Li, L.; et al. Intestinal microbiota metabolism of L-carnitine, a nutrient in red meat, promotes atherosclerosis. Nat Med 2013, 19, 576-585, doi:10.1038/nm.3145.

7. TILLISCH, K.; LABUS, J.; KILPATRICK, L.; JIANG, Z.; STAINS, J.; EBRAT, B.; GUYONNET, D.; LEGRAIN-RASPAUD, S.; TROTIN, B.; NALIBOFF, B.; et al. Consumption of Fermented Milk Product With Probiotic Modulates Brain Activity. Gastroenterology 2013, 144, doi:10.1053/j.gastro.2013.02.043.

8. Shreiner, A.B.; Kao, J.Y.; Young, V.B. The gut microbiome in health and in disease. Curr Opin Gastroenterol 2015, 31, 69-75, doi:10.1097/MOG.0000000000000139.

9. Sinha, R.; Chen, J.; Amir, A.; Vogtmann, E.; Shi, J.; Inman, K.S.; Flores, R.; Sampson, J.; Knight, R.; Chia, N. Collecting Fecal 265 Samples for Microbiome Analyses in Epidemiology Studies. Cancer Epidemiol Biomarkers Prev 2016, 25, 407-416, doi:10.1158/1055-9965.EPI-15-0951.

10. Lucas, C.; Barnich, N.; Nguyen, H.T.T. Microbiota, Inflammation and Colorectal Cancer. International Journal of Molecular Sciences 2017, 18, 1310, doi:10.3390/ijms18061310.

11. Fostira, F.; Thodi, G.; Sandaltzopoulos, R.; Fountzilas, G.; Yannoukakos, D. Mutational spectrum of APC and genotype-phenotype correlations in Greek FAP patients. BMC Cancer 2010, 10, 389, doi:10.1186/1471-2407-10-389.

12. Kostic, A.D.; Chun, E.; Robertson, L.; Glickman, J.N.; Gallini, C.A.; Michaud, M.; Clancy, T.E.; Chung, D.C.; Lochhead, P.; Hold, G.L.; et al. Fusobacterium nucleatum potentiates intestinal tumorigenesis and modulates the tumor immune microenvironment. Cell Host Microbe 2013, 14, 207-215, doi:10.1016/j.chom.2013.07.007.

13. Mehta, R.S.; Nishihara, R.; Cao, Y.; Song, M.; Mima, K.; Qian, Z.R.; Nowak, J.A.; Kosumi, K.; Hamada, T.; Masugi, Y.; et al. Dietary Patterns and Risk of Colorectal Cancer Subtypes Classified by Fusobacterium nucleatum in Tumor Tissue. JAMA Oncol 2017, 3, 921-927, doi:10.1001/jamaoncol.2016.6374.

14. Earley, H.; Lennon, G.; Balfe, A.; Kilcoyne, M.; Clyne, M.; Joshi, L.; Carrington, S.; Martin, S.T.; Coffey, J.C.; Winter, D.C.; et al. 